\title{
Common turbulent signature in sea surface temperature and chlorophyll maps
}

\author{
V. Nieves, ${ }^{1}$ C. Llebot, ${ }^{1}$ A. Turiel,${ }^{1}$ J. Solé, ${ }^{1}$ E. García-Ladona, ${ }^{1}$ M. Estrada, ${ }^{1}$ \\ and D. Blasco ${ }^{1}$ \\ Received 21 June 2007; revised 14 September 2007; accepted 26 October 2007; published 1 December 2007.
}

[1] Oceans and 2D turbulence present similar characteristics, as for instance the dominant role played by eddies in energy and matter transport. However, providing a complete justification of this analogy is difficult, as it requires knowledge of the ocean's dynamic state at different instants and over large scales. Recently, new techniques coming from the Microcanonical Multifractal Formalism have made it possible to infer the streamlines from the analysis of satellite images of some scalar variables. In this paper, we will show that this information is enough to characterize the scaling properties of the energy cascade, which is manifested as a multifractal signature; further, the multifractal signature is obtained at each location in a local basis. Different scalars obtained from satellite measurements such as Sea Surface Temperature or Surface Chlorophyll Concentration present essentially the same multifractal structure, which is interpreted as a consequence of the pervading character of the turbulent advection at the scales of observation. Citation: Nieves, V., C. Llebot, A. Turiel, J. Solé, E. García-Ladona, M. Estrada, and D. Blasco (2007), Common turbulent signature in sea surface temperature and chlorophyll maps, Geophys. Res. Lett., 34, L23602, doi:10.1029/ 2007 GL030823.

\section{Introduction}

[2] The spatial variability of surface ocean tracers is characterized by patchy distributions, resulting from the interplay and balance between advection, diffusion and source and sink processes [Gower et al., 1980; Martin et al., 2002]. Observational evidence of sea surface patterns has stimulated the investigation of which processes dominate and ultimately are the responsible for such complexity. For a tracer such as the Sea Surface Temperature (SST) it has been argued that stirring by the horizontal flow may account for the mesoscale surface signatures seen from satellite images [Abraham and Bowen, 2002]. At such scales ocean dynamics is almost two-dimensional, in quasi-geostrophic equilibrium with low values of the Rossby number (Rossby deformation radii from 10 to $100 \mathrm{~km}$ ). Theoretical arguments on the stirring of tracers by 2-dimensional time-dependent flows have led to explanations of many of the observed features in SST patterns [Waugh et al., 2006]. In the particular case of chlorophyll pigments, some studies on the spatial variability of remote sensing images did not find significant differences between

\footnotetext{
${ }^{1}$ Institut de Ciències del Mar, CSIC, Barcelona, Spain.
}

the spectra of CZCS and SST from AVHRR images [Denman and Abbot, 1994]. This fact led to the conclusion that phytoplankton cells in dynamic areas such as upwelling systems behave largely as passive scalars. Similar trends were found by studying SeaWiFS imagery [Doney et al., 2003], although recent analyses of MODIS images in the Arabian Sea have shown that the variability of SST and Chlorophyll are different [Mahadevan and Campbell, 2002].

[3] Analysis of variance or second order moments poorly characterizes the variability of intermittent fields, so multifractal analysis is much more appropriate [i.e., Lovejoy et $a l ., 2001]$. Very recently the introduction of the Microcanonical Multifractal Formalism (MMF), and in particular the application of singularity analysis to SST images has confirmed the existence of a multifractal structure of surface spatial patterns over a range of scales that includes the mesoscale [Turiel et al., 2005]. One of the most relevant results of this analysis is the observed correspondence between the multifractal patterns in SST and the flow structure at these scales [Isern-Fontanet et al., 2007]. The correspondence is a consequence of the establishment of a multiplicative cascade of the scalar, analogous to the energy cascade of the flow owing to the fully developed turbulence regime [Parisi and Frisch, 1985]. In fact, a passive scalar for which advection dominates should inherit the multifractal character of the flow [Turiel et al., 2005].

[4] In this paper, we will present some new experimental confirmation of the correspondence of the multifractal structure of scalars with the one coming from the flow. We will show that, as for SST, surface Chlorophyll Concentration (CC) maps also exhibit multifractality over a range of scales including the mesoscale. More importantly, the geometrical and statistical structure of the multifractal singularities in CC maps is very close to that of SST images. Further, when different regions are considered the spectra of singularities are coincident. This is evidence that, at the scales resolved by the data, common underlying mechanisms account for the spatial variability of both tracers, and hence their intrinsic dynamics is decoupled from their structure at those scales.

\section{Data Employed in This Study}

[5] For the present work, 47 couples of satellite images derived from Aqua-MODIS ocean color sensor have been processed. MODIS (Moderate Resolution Imaging Spectroradiometer) is a key instrument on board the Terra (EOS $\mathrm{AM}$ ) and Aqua (EOS PM) satellites from NASA. Two different geographical regions were considered. The first one, "North Atlantic Area," extends from 65 to 30 degrees $\mathrm{W}$ in longitude, and from 45 to 60 degrees $\mathrm{N}$ in latitude. The 
second region, "South Africa Area," extends from 5 to 25 degrees $\mathrm{E}$ in longitude and from 40 to 10 degrees $\mathrm{S}$ in latitude. North Atlantic Area includes part of the Labrador peninsula and of East and West Greenland currents; in addition, on its Southeast side it encompasses a portion of the limit of the North Atlantic gyre delimited by the North Atlantic Current, which transports warm waters from the Gulf Stream poleward. South Africa Area covers the Benguela current, which is the eastern boundary current of the South Atlantic subtropical gyre advecting cool waters to the tropics; it is a region characterized by a richly productive marine ecosystem due to an important upwelling coastal system (J. Gyorya, The Benguela current, available at http:// oceancurrents.rsmas.miami.edu/atlantic/benguela.html, 2001). At each time and location, Aqua-MODIS images of two different types have been considered: chlorophyll concentration (CC) and daytime sea surface temperature (SST) images, spatially averaged (Level 3 products) over a $4 \times 4 \mathrm{~km}^{2}$ pixel grid (resolution at the equator). Processing details can be found at http://oceancolor.gsfc.nasa.gov/ DOCS/modis_sst/.

[6] We have processed daily-derived SST images only, in order to have SST and CC images as simultaneous as possible. The images are affected by noise and are spatially discontinuous due to several effects, including cloud cover, aerosol corruption, orbital gaps and aberrant values in the SST/CC algorithms. A single image can be the result of the merging of two or more satellite passes, so evidencing that singularity analysis serves to isolate a universal mechanism, which partially diminishes errors and data loss; however, the merging process also blurs to some extent the sharpest transitions and tends to produce slightly mislocated singularity fronts. This blurring does not equally affect both types of images, so any image comparison should be based on regional comparisons instead of point to point comparisons. For each area, we tried to generate two ensembles with similar numbers of coupled SST-CC images, selecting those images with as few lost data as possible. We thus chose twenty-four images corresponding to South Africa Area, captured during 2005 and twenty-three images from the North Atlantic, captured in a period extending from 2003 to 2006 and almost evenly representing all seasons.

\section{Singularity Analysis}

[7] Recently [Turiel et al., 2005; Isern-Fontanet et al., 2007], a new approach to turbulence analysis, based on the explicit geometrical organization of the flows, has arisen. This approach intends to characterize local scaling properties at each point (geometrical or microcanonical approach), instead of global properties of the statistical ensemble (statistical or canonical approach).

[8] In MMF, the key concept is that of the singularity exponent: each point in the flow can be characterized by a singularity exponent, which is a dimensionless, scaleinvariant measure of the regularity or irregularity of the flow in the neighborhood of that point [Turiel et al., 2005]. The local singularity exponent $h(\mathbf{x})$ of a given field $s(\mathbf{x})$ at the point $\mathbf{x}$ can be obtained, for any vector $\mathbf{r}$ small enough, from the following scaling

$$
\frac{1}{r}|s(\mathbf{x}+\mathbf{r})-s(\mathbf{x})| \sim r^{h(\mathbf{x})}
$$

where $r$ is a given resolution scale. The singularity exponent $h(\mathbf{x})$ is a measure of the degree of regularity of $s$ at $\mathbf{x}$ : negative values correspond to sharp transitions (brighter colors in Figures 1 and 2), while positive exponents represent smooth, regular structures (darker colors in the Figures 1 and 2). The values of $h(\mathbf{x})$ typically fall in the range $(-1,2)$ [Turiel et al., 2005; Isern-Fontanet et al., 2007]. Expressions such as equation (1) are generically known as Singularity Analysis for the function $s(\mathbf{x})$ [Turiel et al., 2005]. The scaling properties of the velocity field are in general difficult to quantify, but they can be connected to the scaling properties of related variables much more accessible and easier to observe. A typical case is that of scalar variables. If a multifractal hierarchy sensu Parisi \& Frisch [Parisi and Frisch, 1985] is evidenced for a given scalar, we will accept that its most plausible origin is the turbulent flow driving the scalar. That is, the flow transmits its singularity exponents to any scalar for which advection is strong enough [Abraham and Bowen, 2002; Turiel et al., 2005]. As a consequence, the obtaining of singularity exponents starting from any scalar quantity reveals properties of the flow motion. Experimental evidences [Abraham and Bowen, 2002; Turiel et al., 2005] indicate that singularities are advected by the flow. Hence, the spatial distribution of singularities should trace the instantaneous streamlines of the motion and therefore, the velocity field can in principle be retrieved [Turiel et al., 2005].

[9] Equation (1) is very appealing and easy to relate to the properties of the flow. However, long-range correlations can mask the largest singularity exponents [Turiel et al., 2005]. Thus, $s(\mathbf{x})$ has to be filtered. Continuous wavelet transforms, defined as projections of the signal at different resolutions, are appropriated to filter multiscaling functions without disturbing scale invariance properties. In addition, it is convenient to work with the gradient modulus of the signal, $|\nabla s|(\mathbf{x})$, to improve the spatial resolution of the results [Turiel et al., 2006]. Equation (1) has a wavelettransformed counterpart:

$$
T_{\Psi}|\nabla s|(\mathbf{x}, r) \sim r^{h(\mathbf{x})}
$$

where $T_{\Psi}|\nabla s|(\mathbf{x}, r)$ is the wavelet projection of $|\nabla s|$ over the wavelet $\Psi$ at the point $\mathbf{x}$ and the scale $r>0$, defined as:

$$
T_{\Psi}|\nabla s|(\mathbf{x}, r) \equiv \int d \mathbf{x}^{\prime}|\nabla s|\left(\mathbf{x}^{\prime}\right) \frac{1}{r^{2}} \Psi\left(\frac{\mathbf{x}-\mathbf{x}^{\prime}}{r}\right) .
$$

(see, for instance, Turiel et al. [2005]). A family of wavelets has been shown [Turiel et al., 2005; Isern-Fontanet et al., 2007] to be the most efficient to attain good discrimination and resolution capabilities, the Lorentzian family: $\Psi \gamma(\mathbf{x})=$ $1 /\left(1+|\mathbf{x}|^{2}\right)^{\gamma}$, for $\gamma \geq 1$. We have used a numerical implementation of $\Psi_{\gamma=1}$ for the analyses shown in this paper.

\section{Validity of MMF for SST and CC images}

[10] An extensive presentation of MMF is given by IsernFontanet et al. [2007]. Let us summarize here the three conditions for MMF.

[11] (1) Each fractal component is scale-invariant: at any point $\mathbf{x}$, a well-defined local power-law scaling of exponent $h(\mathbf{x})$, according to Equation 2, must be observed for a large enough range of scales $r$. We have verified that the equation 

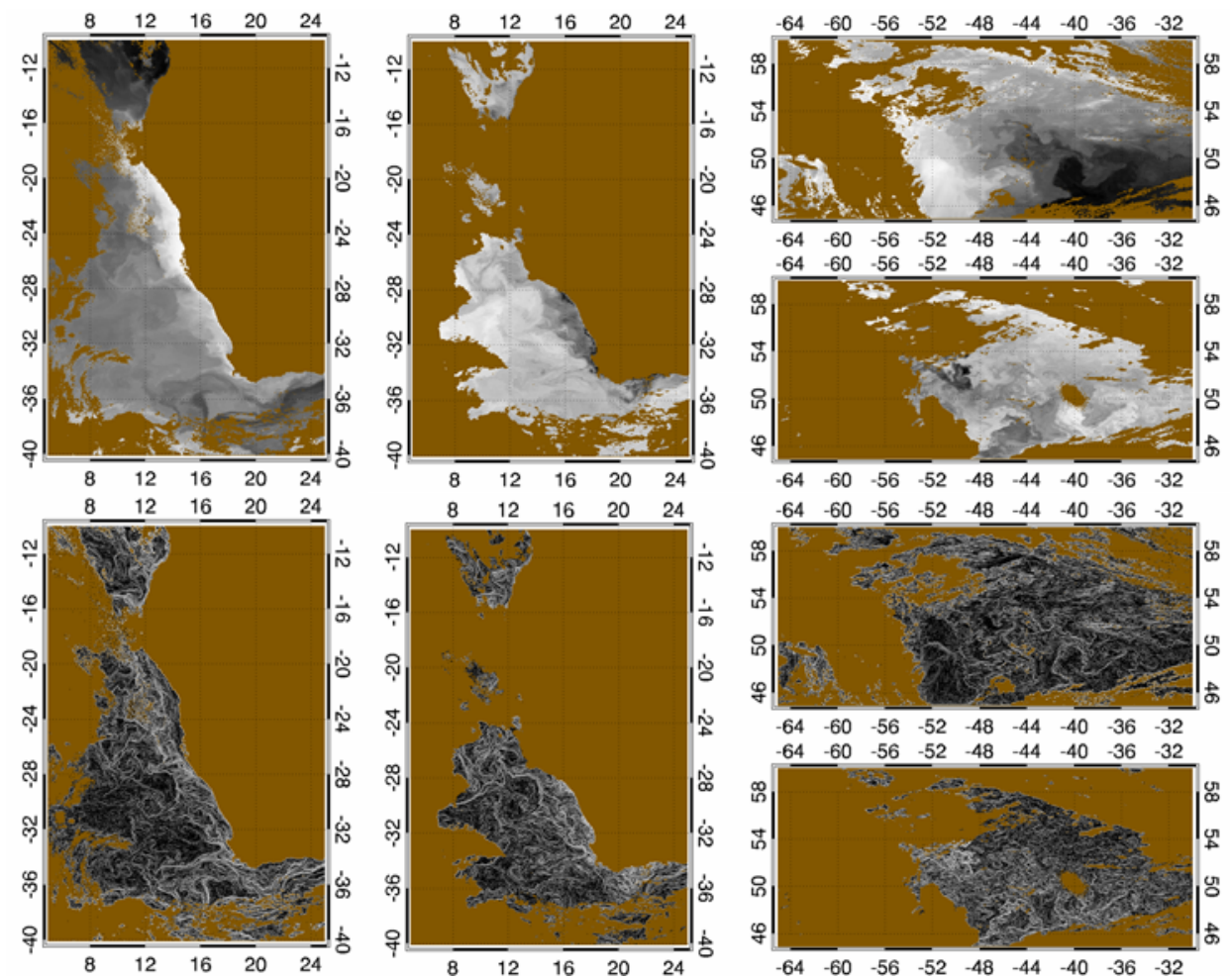

Figure 1. Figures from left to right and from top to bottom. Vertical maps: data obtained in the South Africa Area in April 28, 2005, which correspond to SST and CC; singularity exponents associated to SST and CC images for the same area and period. Horizontal maps: images from North Atlantic Area in April 25, 2006, showing both scalars as well as their respective singularity exponents.

holds with good accuracy (using the criterion introduced by Isern-Fontanet et al. [2007]) up to 149 pixels $(596 \mathrm{~km})$ for both SST and CC.

[12] (2) The whole multifractal hierarchy is scaleinvariant: the probability distributions of singularities obtained at different scales $r$ lead to the same singularity spectrum curve $D(h)$. From the empirical histogram $\rho_{r}(h)$ obtained at the scale $r$ we can obtain the singularity spectrum [Turiel et al., 2006; Isern-Fontanet et al., 2007], according to

$$
D(h)=d-\frac{\log \left(\frac{\rho_{r}(h)}{\max \left\{\rho_{r}(h)\right\}}\right)}{\log r}
$$

[13] We have evaluated the histograms at the original resolution and after a reduction by a factor 4 (curves not shown). We conclude that this property is well satisfied by SST and CC images.

[14] (3) The curve $D(h)$ is convex, which implies that the cascade is actually developed in the flow [see Isern-Fontanet et al., 2007]. The curves shown in Figure 3 are convex, so validating this condition.

[15] So, both SST and CC are multifractal signals in the sense of MMF. We hypothesize that this structure is a consequence of oceanic turbulence.

\section{Comparison Between SST and CC Multifractal Structures}

[16] In Figure 1 we show a couple of SST-CC Aqua MODIS images and their associated singularity exponents according to MMF in the South Africa Area. Singularity analysis puts in evidence main frontal regions with an unprecedented quality but also many subtle non-trivial structures, revealing the presence of eddies and flow lines that passed almost unnoticed in the original images. Remarkably enough, there is a good visual correspondence between the features detected in SST and CC, reinforcing the assumption of a common dynamical origin for the two multifractal structures.

[17] The cascading processes associated to each type of scalar can be compared by studying the statistics of the corresponding singularity spectra; in this case, MMF allows a derivation of the structure of the turbulent cascade with relatively reduced datasets leading to experimental values of $D(h)$ with a typical uncertainty of about 0.05 [Turiel et al., 2006]. As shown in Figure 3 a good correspondence of the turbulent cascades is found over the two regions under study for both scalars. All singularity spectra are defined on about the same range of values, and differences in $D(h)$ are less than 0.1 , compatible with the experimental uncertainty. For both types of data, SST and CC, the good correspondence of the singularity spectra obtained for two distant geographical regions indicates that the underlying mechanisms which gave rise to the multifractal structures should be similar.

[18] We arrive at the same conclusion when comparing the singularity spectra of SST and CC. Although theoretically granted if advection induces the multifractal structure, a point-by-point coincidence between singularities is hard to verify on data, as singularities appear slightly mislocated due to differences in acquisition processes and the own spatial and functional uncertainty of the singularity analysis. 

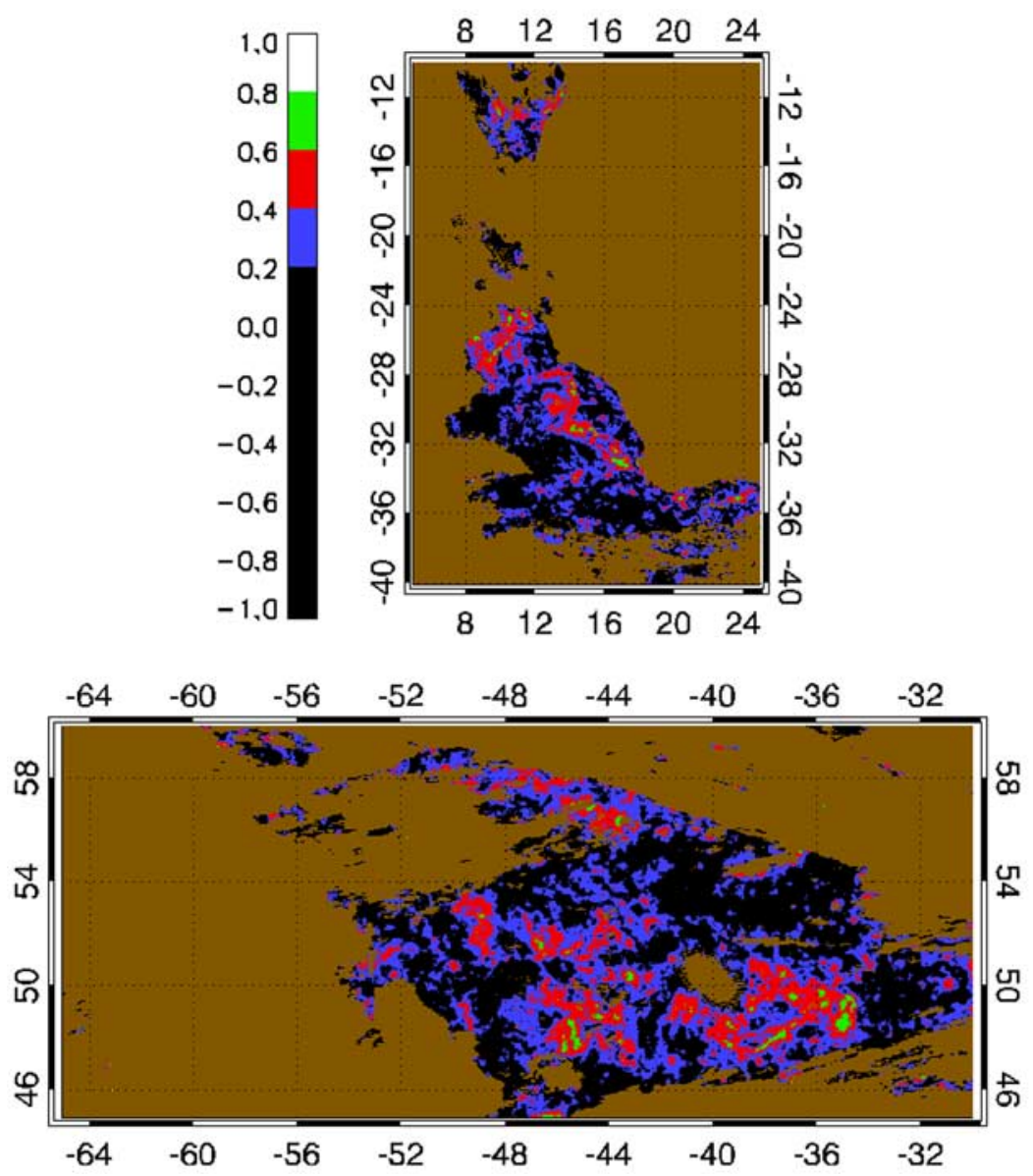

Figure 2. Local correlation coefficients between SST and CC singularity exponents shown in Figure 1.

We will thus define a local degree of closeness between the singularity maps. The proposed measure is given by a weighted local regression coefficient. Our measure of closeness between $h_{T}$ and $h_{C}$ is given by $\rho_{T C}(\mathbf{x})=\frac{\sigma_{T C}(\mathbf{x})}{\sigma_{T}(\mathbf{x}) \sigma_{C}(\mathbf{x})}$, where $\sigma_{T}^{2}(\mathbf{x})=\left\langle h_{T}^{2}\right\rangle_{\mathbf{x}}-\left\langle h_{T}\right\rangle_{\mathbf{x}}^{2}, \sigma_{C}^{2}(\mathbf{x})=\left\langle h_{C}^{2}\right\rangle_{\mathbf{x}}-\left\langle h_{C}\right\rangle_{\mathbf{x}}^{2}$ and $\sigma T C(\mathbf{x})=\left\langle h_{T} h_{C}\right\rangle_{\mathbf{x}}-\left\langle h_{h T}\right\rangle_{\mathbf{x}}\left\langle_{h C}\right\rangle_{\mathbf{x}}$.

[19] At any point $\mathbf{x}$, we will define the weighted average of any function of $h_{T}$ and $h_{C}, F\left(h_{T}, h_{C}\right)$, in the following way:

$$
\left\langle F\left(h_{T}, h_{C}\right)\right\rangle_{\mathbf{x}} \equiv \frac{1}{N_{\mathbf{x}}} \sum_{\mathbf{x}^{\prime}} \frac{F\left(h_{T}\left(\mathbf{x}^{\prime}\right), h_{C}\left(\mathbf{x}^{\prime}\right)\right)}{b\left(h_{T}\left(\mathbf{x}^{\prime}\right)\right) b\left(h_{C}\left(\mathbf{x}^{\prime}\right)\right)} w\left(\mathbf{x}-\mathbf{x}^{\prime}\right)
$$

where $N_{\mathbf{x}}=\sum_{\mathbf{x}^{\prime}} \frac{1}{b\left(h_{T}\left(\mathbf{x}^{\prime}\right)\right) b\left(h_{C}\left(\mathbf{x}^{\prime}\right)\right)} w\left(\mathbf{x}-\mathbf{x}^{\prime}\right)$ is a normalization constant which takes into account the points $\mathbf{x}^{\prime}$ actually available; $b(h)$ is a positive function measuring the uncertainty in the value of $h$; and $w(\mathbf{x})$ is an appropriate weighting function, used to define the relative importance of the points on the region centered around $\mathbf{x}$. A reasonable choice for $\mathrm{w}(\mathbf{x})$ is an isotropic function (that is, only depending on $|\mathbf{x}|$ ) that decays with distance. Good weighting functions are those in the scale-invariant family, namely: $w(\mathbf{x})=\frac{1}{|\mathbf{x}| \alpha}$ for $\alpha=$ 2 in our case. With respect to the $h$-uncertainty, measured by the function $b(h)$, we have used an heuristic estimate, based on the observations reported in singularity analysis of
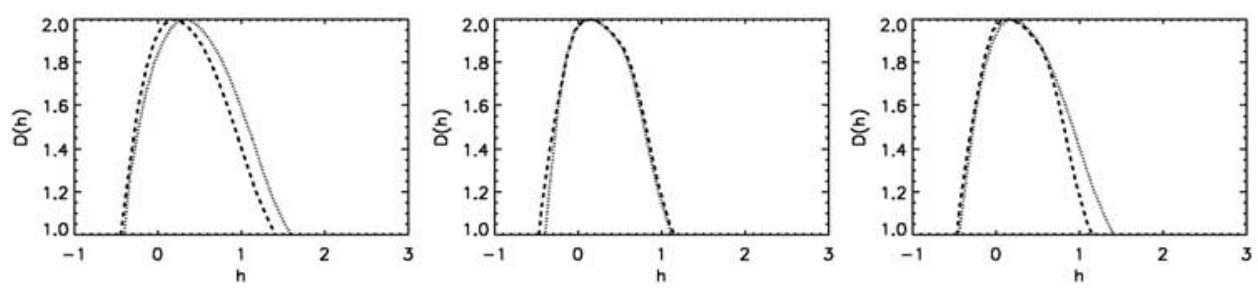

Figure 3. (left) Comparison of the singularity spectra $D(h)$ derived from SST in the North Atlantic (dotted line) and the South Africa Area (dashed line). (middle) The same for the spectra derived from CC, with identical conclusions. (right) Comparison of singularity spectra obtained from the whole dataset of SST images (dotted line) and CC images (dashed line). 
images of different types [Turiel et al., 2005]. We have used $b(h)=0.1$ for $h \leq 0.1$ and $b(h)=h$ for $h>0.1$.

[20] As the distributions of $h_{T}$ and $h_{C}$ are identical (see Figure 3), the only possible linear transformation is $h_{T}=h_{C}$. Hence, when $\rho_{T C}$ is close to 1 , both types of singularity exponents can be considered as identical over that region. Due to all the processing effects, the coincidence is not perfect, although remarkable. Looking at the maps of local regression coefficients, Figure 2, we can confirm the closeness between the maps of SST and CC exponents, specially over free areas. This, together with the coincidence of spectra, evidences the existence of a similar turbulent-like cascading process acting on SST and CC. We conclude that we are certainly extracting information on a dominant mechanism, the advective mode of the flow acting on both scalars.

\section{Conclusions}

[21] The evidence shown in this paper can be summarized by saying that over a rather broad range of scales, including the mesoscale, ocean turbulence has a strong influence on the geometrical arrangement of tracers of very different nature. This is supported by the remarkable similarity between the spectra of singularity exponents over distinct regions for both tracers. Then the spatial arrangement for the most singular exponents, which basically identify the places with strong enough advection of the scalars, gives information on the streamlines. This has two relevant consequences. First, as an added value we are in fact inferring a large amount of instantaneous information on the dynamics of ocean flows. The obtaining of singularity exponents from images may allow to determine a first estimate of the instantaneous field of sea surface currents [Turiel et al., 2005]. Systematic and extensive re-analysis of recent satellite imagery data bases, covering up to twenty years of SST and ten years of CC, opens the way to assess at a global scale the effects of climate variability and change over the past two decades. Furthermore, the local correspondence of SST and CC singularities due to the existence of a common cascade process may make it possible to use SST data (which are more abundant than CC data) for helping the inference of reasonable distributions of $\mathrm{CC}$ at those locations where $\mathrm{CC}$ data are lacking. On the other hand analyzing the areas in which the correspondence of both tracers fails may help to identify where the intrinsic dynamics of the tracer is in competition with flow advection which in turn would serve, in the case of $\mathrm{CC}$, to improve our knowledge on ocean phytoplankton communities and improve our understanding of biological processes.

[22] Acknowledgments. A. Turiel is contracted under the Ramon y Cajal program by the Spanish Ministry of Education. V. Nieves is supported by a Ph.D. grant funded by MERSEA. C. Llebot is supported by a Ph.D. grant funded by CSIC, J. Solé is supported by a post-doc grant funded by EU Streps Project SEEDS. This work is a contribution to the MERSEA project. Partial support from the European Commission under contract SIP3-CT-2003-502885 is gratefully acknowledged. This work is also a contribution to the Spanish projects ESEOO (VEM2003- 20577-C14-10), MIDAS-4 (ESP2005-06823-C05-1) and OCEANTECH (PIF-2006).

\section{References}

Abraham, E., and M. Bowen (2002), Chaotic stirring by a mesoscale surface-ocean flow, Chaos, 12(2), 373-381, doi:10.1063/1.1481615.

Denman, K., and M. R. Abbot (1994), Time scales of pattern evolution from cross-spectrum analysis of advanced very high resolution radiometer and coastal zone color scanner imagery, J. Geophys. Res., 99(C4), 7433-7442.

Doney, S. C., D. M. Glover, S. J. McCue, and M. Fuentes (2003), Mesoscale variability of Sea-viewing Wide Field-of-view Sensor (SeaWiFS) satellite ocean color: Global patterns and spatial scales, J. Geophys. Res., 108(C2), 3024, doi:10.1029/2001JC000843.

Gower, J. F. R., K. L. Denman, and R. J. Holyer (1980), Phytoplankton patchiness indicates the fluctuation spectrum of mesoscale oceanic structure, Nature, 288, 157-159.

Isern-Fontanet, J., A. Turiel, E. García-Ladona, and J. Font (2007), Microcanonical multifractal formalism: Application to the estimation of ocean surface velocities, J. Geophys. Res., 112, C05024, doi:10.1029/ 2006JC003878.

Lovejoy, S., D. Schertzer, Y. Tessier, and H. Gaonach (2001), Multifractals and resolution-independent remote sensing algorithms: The example of ocean colour, Int. J. Remote Sens., 22(7), 1191-1234.

Mahadevan, A., and J. W. Campbell (2002), Biogeochemical patchiness at the sea surface, Geophys. Res. Lett., 29(19), 1926, doi:10.1029/ 2001 GL014116.

Martin, A. P., K. J. Richards, A. Bracco, and A. Provenzale (2002), Patchy productivity in the open ocean, Global Biogeochem. Cycles, 16(2), 1025, doi:10.1029/2001GB001449.

Parisi, G., and U. Frisch (1985), On the singularity structure of fully developed turbulence, in Turbulence and Predictability in Geophysical Fluid Dynamics, Proc. Int. School Phys. E. Fermi, vol. 88, edited by M. Ghil, R. Benzi, and G. Parisi, pp. 84-87, North-Holland, Amsterdam. Turiel, A., J. Isern-Fontanet, E. García-Ladona, and J. Font (2005), Multifractal method for the instantaneous evaluation of the stream function in geophysical flows, Phys. Rev. Lett., 95(10), 104502, doi:10.1103/PhysRevLett.95.104502.

Turiel, A., C. Pérez-Vicente, and J. Grazzini (2006), Numerical methods for the estimation of multifractal singularity spectra on sampled data: A comparative study, J. Comput. Phys., 216(1), 362-390.

Waugh, D., E. Abraham, and M. Bowen (2006), Spatial variations of stirring in the surface ocean: A case study of the Tasman sea, J. Phys. Oceanogr., 36(3), 526-542.

D. Blasco, M. Estrada, E. García-Ladona, C. Llebot, V. Nieves, J. Solé, and A. Turiel, Institut de Ciències del Mar, CSIC, Passeig Marítim de la Barceloneta 37-49, E-08003 Barcelona, Spain. (blasco@icm.csic.es; marta@icm.csic.es; emilio@icm.csic.es; llebot@icm.csic.es; vnieves@icm. csic.es; jsole@icm.csic.es; turiel@icm.csic.es) 